Research Article

\title{
Dynamic Cross-Correlations Analysis on Economic Policy Uncertainty and US Dollar Exchange Rate: AMF-DCCA Perspective
}

\author{
Ruwei Zhao' and Yian Cui ${ }^{2}{ }^{2}$ \\ ${ }^{1}$ School of Business, Jiangnan University, Wuxi, Jiangsu 214122, China \\ ${ }^{2}$ Research Institute, Shenzhen Stock Exchange, Shenzhen 518038, China \\ Correspondence should be addressed to Yian Cui; yacui@szse.cn
}

Received 11 October 2020; Revised 7 December 2020; Accepted 9 January 2021; Published 30 January 2021

Academic Editor: Giancarlo Consolo

Copyright (c) 2021 Ruwei Zhao and Yian Cui. This is an open access article distributed under the Creative Commons Attribution License, which permits unrestricted use, distribution, and reproduction in any medium, provided the original work is properly cited.

In this paper, we employ the multifractal detrended cross-correlation analysis (MF-DCCA) as the measurement instrument for the dynamic cross-correlation inspection between US economic policy uncertainty (EPU) index and US dollar exchange rate return (Ret). By calculating the cross-correlation statistics, we find mild acceptance of cross-correlation between EPU and Ret qualitatively. With further application of MF-DCCA methodology, we find strong power law cross-correlation existence within all scaling orders. Also, apparent persistence of cross-correlation has been discovered with significant Hurst exponents of all orders. Besides, we find that long-term cross-correlation demonstrates more persistence and higher degree of multifractality than those in the short term. Finally, we utilize the rolling window and binominal measurement analysis as revisits of the model. The results are consistent with model statements.

\section{Introduction}

It is well documented that macro factors demonstrate powerful influence in pricing financial assets, such as stocks and bonds [1-3]. However, few studies concentrate on the exchange market. Also, to have a better observation of economic policy fluctuation in a quantitative way, Baker et al. [4] develop the novel economic policy uncertainty index with the retrieval of mainstream newspapers, which is widely employed in the financial academic field [5-22]. With this view, we connect the prevailing economic policy uncertainty index with US dollar, the world's largest trading currency, to check if US macro policy adjustment would shed light on the fluctuation of US dollar exchange rate. Due to the introduction of Fractal Market Hypothesis (FMH) suggested by Mandelbrot and Van Ness [23], many researchers are inclined to employ fractal analysis methodology, such as multifractal detrended cross-correlation analysis (MF-DCCA), as the statistical instrument for the fractal characteristics discovery between nonstationary financial time series [24-32]. Zhang et al. [25] carried out a study regarding the correlation between media news and stock market index return with MF-DCCA approach. They found quantitative evidence for the cross-correlation multifractality existence between media news and SSE 50 index return. They further conducted the rolling window analysis, and the results show that scaling exponents are all above critical values, showing strong evidence of multifractality persistence between media news and index return. Zhou et al. [28] took an information content investigation of financial derivatives in China market. With MF-DCCA approach, they found that put-call ratio demonstrates antipersistent cross-correlation with 50 ETF return. Meanwhile, for the option-to-stock volume ratio, no significant cross-correlation was detected.

In this study, we employ the economic policy uncertainty index, constructed through semantic analysis techniques with hundreds of mainstream newspapers, as the representative of national economic policy fluctuation. Many prior studies have confirmed the applicability 
of the economic policy uncertainty index and MF-DCCA approach [5-22, 24-32]. With this view, we utilize the MF-DCCA as a vehicle to check the existence of multifractal cross-correlation between US economic policy uncertainty index and US dollar exchange rate index return. We calculate the fluctuation function and find widespread power law cross-correlation existence. Moreover, the cross-correlations between series demonstrate strong persistence with significant exponents of all scaling orders. In addition, we calculate the time turning point and break the whole-time length into short-term and long-term periods. We find that long-term crosscorrelation performs much better in persistence and multifractality compared with those in the short term. In the end, we revisit the MF-DCCA model with the rolling window and binominal measurement analysis. The results confirm series multifractality with the qualified exponents and close distances between arithmetic average and crosscorrelation exponents.

Our study contributes to the existing literature from three perspectives. First, we utilize the novel US economic policy uncertainty index as the measurement for the US economic policy fluctuation and concatenate it with the US dollar exchange rate index with MF-DCCA methodology. Particularly, we find strong power law cross-correlation existence between US economic policy fluctuation and US dollar exchange rate return within all scaling orders. Also, the cross-correlations demonstrate reliable persistence with significant exponents. With this view, our empirical findings were consistent with the financial studies associated with MF-DCCA [24-32]. Second, our study originates from the exchange market perspective, providing deeper insight into the evolution dynamics of the US dollar exchange rate. Third, as the world's largest trading currency, our findings would provide valuable suggestions for investor's risk management in the US dollar exchange market by hedging with economic fluctuation.

The rest of this paper is organized as follows: Section 2 describes the data. Section 3 illustrates the multifractal crosscorrelation methodology. Section 4 demonstrates the empirical results. Section 5 concludes the paper.

\section{Literature Review}

In this study, we investigate whether multifractal crosscorrelation between economic policy uncertainty and US dollar index exists with the application of multifractal detrended cross-correlation analysis (MF-DCCA). With this view, we develop the review from the impact of economic policy uncertainty on asset pricing and the application of MF-DCCA in financial time series perspectives.

2.1. Economic Policy Uncertainty. Many studies have revealed that macro factors, such as changes in economic policies, present significant power in pricing financial assets. Bhamra et al. [3] and Chen [2] provided theoretical models embedded with economic uncertainty to answer the credit spread puzzles. However, to have a general measurement of economic policy uncertainty in a quantitative way, Baker et al. [4] constructed a novel index of economic policy uncertainty based on the analysis of newspaper coverage frequency, which is widely employed by financial researchers. Lee et al. [5] connected the China economic policy uncertainty index with US household portfolio changes. They found that US household would decrease stock holdings with increasing China economic policy uncertainty, especially for the states with more exports to China. Attig et al. [6] studied the relation between economic policy uncertainty index and company dividend payout policy internationally. The empirical findings suggest that corporate executives prefer to distribute more dividends to shareholders in the higher EPU times. Yang et al. [14] investigated whether economic policy uncertainty index holds prediction for the excess return. They found that the greater dispersion of economic policy uncertainty index would result in a higher rate of excess return in China's stock market. Hsieh and Nguyen [11] carried out a study on economic policy uncertainty index and illiquidity return premium. They found that the premium between illiquidity and liquidity portfolios would be larger when the economic policy uncertainty index begins to have an upward trend.

2.2. Multifractal Detrended Cross-Correlation Analysis. Extensive studies contribute to methodology development of nonstationary time series analysis. Kantelhardt et al. [33] proposed multifractal detrended fluctuation analysis (MFDFA) for the examination of nonstationary time series, extending prior detrended fluctuation analysis (DFA) proposed by Peng et al. [34, 35]. Podobnik and Stanley [36] put forward detrended cross-correlation analysis (DCCA) enabling cross-correlation investigation between two nonstationary time series. However, detrended cross-correlation analysis is from the single fractal perspective, resulting in potential information loss of the time series. To have deeper insight into two nonstationary time series, Zhou [37] advanced the prevailing multifractal detrended cross-correlation analysis (MF-DCCA) by combining MFDFA with DCCA. Ruan et al. [24] studied the multifractal characters among China's agricultural futures returns. They found credible multifractality existences among soybean, soymeal, and soyoil futures returns. Cai et al. [29] studied the cross-correlations between crude oil price and implied volatility indices. They found that the cross-correlation multifractalities are ubiquitous between series. Alaoui et al. [30] studied the cross-correlation between Bitcoin price and volume. With the application of MF-DCCA, they found adequate evidence supporting the cross-correlation multifractality between Bitcoin price and volume. Wang et al. [31] examined the cross-correlation between crude oil and agriculture futures under the shock of COVID-19. They found that the persistence between series is greatly enhanced after the involvement of COVID19 and the cross-correlation between crude oil and sugar future presents strongest multifractality. 


\section{Data Description}

We obtain the daily data of US EPU index by downloading it directly from the website (http://www.policyuncertainty. com/us_monthly.html). US daily EPU index is based on the article archives from Access World News NewsBank service. The Access World News NewsBank database contains over 1000 newspapers from widely known to small local newspapers across US. The measure for this index is the number of articles that contain at least one term from each of 3 sets of terms. The first set is economic or economy. The second is uncertain or uncertainty. The third set is legislation or deficit or regulation or congress or federal reserve or white house. We derive US dollar index as proxy for US foreign exchange market from Yahoo Finance. The sample periods of US daily EPU index and US dollar index are from 1 January 1985 to 8 October 2020.

In addition, we employ two log values as proxies for the cross-correlation inspection between EPU and US dollar index. The calculation processes are as follows:

$$
\begin{aligned}
\operatorname{Ret}_{t} & =\ln \left(\frac{p_{t}}{p_{t-1}}\right), \\
E P U_{t} & =\ln \left(e p u_{t}\right),
\end{aligned}
$$

where $P_{t}$ is the closing price of US dollar exchange rate at day $t$ and $\mathrm{EPU}_{\mathrm{t}}$ is the daily value of EPU index. Table 1 reports the descriptive statistics of EPU index and US dollar exchange rate return. As we can see in Table 1, the means of Ret and EPU ( 0.00 and 4.39) are smaller than the medians $(0.00$ and 4.40), showing a left-skew character, which is consistent with negative numbers of skewness $(-0.08$ and -0.13$)$. For the kurtosis, the two series demonstrate sharp peak characteristics with values greater than 3 (5.22 and 3.39). In addition, we calculate the Jarque-Bera coefficients within each series to check the normality existence. We can find that both series present strong rejections of the normal distributions with coefficients significant at $1 \%$ levels $\left(1878.42^{* * *}\right.$ and $\left.83.08^{* * *}\right)$. Also, the standard deviation of EPU (0.68) is much larger than that of Ret (0.01), indicating a higher level of volatility.

\section{Methodology}

In this section, we employ the prevailing MF-DCCA approach to check whether multifractality exists between the US dollar exchange rate and US economic policy uncertainty. We first utilize the methodology proposed by Podobnik and Stanley [36] to have a qualitative cross-correlation examination between series. Second, we apply the MF-DCCA approach to check if the correlations hold multifractality with the changes of scaling parameters.

4.1. Cross-Correlation Test. Before the MF-DCCA inspection, we employ a cross-correlation statistics proposed by Podobnik and Stanley [36] to have a qualitative cross-correlation check between US dollar exchange rate return and US economic policy uncertainty index. The cross-correlation
TABLE 1: Descriptive statistics of Ret and EPU.

\begin{tabular}{lcc}
\hline Variable & Ret & EPU \\
\hline Mean & 0.00 & 4.39 \\
Median & 0.00 & 4.40 \\
Standard deviation & 0.01 & 0.68 \\
Max & 0.03 & 6.69 \\
Min & -0.04 & 1.20 \\
Skewness & -0.08 & -0.13 \\
Kurtosis & 5.22 & 3.39 \\
Jarque-Bera & $1878.42^{* * *}$ & $83.08^{* * *}$ \\
N & 9129 & 9129 \\
\hline
\end{tabular}

The Ret and EPU terms are short for the daily US dollar exchange rate return and the US economic policy uncertainty index. $N$ refers to the number of observations. ${ }^{* * *}$ Statistical significance at $1 \%$ level.

statistics is constructed through two steps. Firstly, we need to have a cross-correlation indicator. The indicator $C_{i}$ is created as follows:

$$
C_{i}=\frac{\sum_{k=i+1}^{N} x_{k} y_{k-i}}{\sqrt{\sum_{k=1}^{N} x_{k}^{2} \sum_{k=1}^{N} y_{k}^{2}}},
$$

where $\left\{x_{k}\right\}$ and $\left\{y_{k}\right\}$ are the two time series with equal lengths of $\mathrm{N}$.

Secondly, we calculate the cross-correlation statistics with the involvement of prior indicator $C_{i}$. The statistics construction process is as shown in the following equation:

$$
Q_{\mathrm{cc}}(m)=N^{2} \sum_{i=1}^{m} \frac{C_{i}^{2}}{N-i},
$$

where $C_{i}$ is the cross-correlation indicator, $N$ is the number of observations, and $m$ works as the degree of freedom. The cross-correlation statistics $Q_{\mathrm{cc}}(m)$ follow the $\chi^{2}(m)$ distribution with $m$ degrees of freedom. The null hypothesis of the cross-correlation test proposed by Podobnik and Stanley [36] states that the cross-correlation indicator $C_{i}$ demonstrates no significant difference from zero. With this view, we compare the cross-correlation statistics $Q_{c c}(m)$ and chi-square critical value $\chi^{2}(m)$ with $m$ degrees of freedom to see whether the value of the cross-correlation statistics $Q_{c c}(m)$ is greater than that of the chi-square critical value $\chi^{2}(m)$. If so, the null hypothesis would be rejected and a reliable cross-correlation between two time series can be confirmed in a statistical way.

4.2. MF-DCCA. Zhou [37] proposed the multifractal crosscorrelation analysis (MF-DCCA) for the cross-correlation inspection between time series, which is widely employed in the academic field. With this view, we employ the prevailing MF-DCCA methodology as a vehicle to check if the multifractal cross-correlation exists between the return of US dollar exchange rate and US economic policy uncertainty index. The MF-DCCA approach is derived from the detrended fluctuation analysis. The five detailed construction steps are as follows. 
Step 1. We have two equal-length time series $\left\{x_{k}\right\}$ and $\left\{y_{k}\right\}$ as the indicators of the US dollar exchange rate return and US economic policy uncertainty index, where $k$ is from 1 to $\mathrm{N}$. N denotes the number of the total observations. Afterwards, two other detrended profiles $X(i)$ and $Y(i)$ are created by original time series $\left\{x_{k}\right\}$ and $\left\{y_{k}\right\}$, where $i$ is from 1 to $N$, respectively. The detailed calculation process is as follows:

$$
\begin{aligned}
& X(i)=\sum_{k=1}^{i}\left(x_{k}-\bar{x}\right), \\
& Y(i)=\sum_{k=1}^{i}\left(y_{k}-\bar{y}\right),
\end{aligned}
$$

where $\bar{x}$ and $\bar{y}$ are arithmetic average values of the time series $\left\{x_{k}\right\}$ and $\left\{y_{k}\right\}$. With this view, it is easy to find that $X$ $(N)=Y(N)=0$.

Step 2. We further divide two detrended profiles $X(i)$ and $Y$ (i) into $N_{s}$ nonoverlapping segments. Each segment is a separate time series with $s$ observations. The calculation process of length interval $N_{s}$ is as follows:

$$
N_{s}=\operatorname{int}\left(\frac{N}{s}\right)
$$

where $N$ refers to the number of total observations. "int" is the symbol of integer function, which accounts for the collection of the maximum integer toward the real number. In addition, the total number $N$ sometimes cannot be divided completely by scale $s$ without any remainder. This would generate a short part segment ignorance at the end of each profile. To maximize the value of the entire series, we regenerate $N_{s}$ segments from the end to the top of the profile. As a result, each profile holds two $N_{s}$ segments after this procedure.

Step 3. To acquire the local trend of each of the two $N_{s}$ segments, we perform a polynomial fitness check with each segment. As a result, the variance of each segment $v$ is constructed as follows:

If $v=1,2,3, \ldots, N_{s}$, the variance of segment $\nu, F^{2}(\nu, s)$, is as follows:

$$
F^{2}(\nu, s)=\frac{1}{s} \sum_{i=1}^{s}\left|X((\nu-1) s+i)-p_{v}^{n}(i)\right| \cdot\left|Y((\nu-1) s+i)-p_{v}^{n}(i)\right|
$$

If $v=N_{s}+1, N_{s}+2, N_{s}+3, \ldots, 2 N_{s}$, the variance of segment $\nu, F^{2}(\nu, s)$, is as follows:

$$
F^{2}(\nu, s)=\frac{1}{s} \sum_{i=1}^{s}\left|X\left(N-\left(\nu-N_{s}\right) s+i\right)-p_{v}^{n}(i)\right| \cdot\left|Y\left(N-\left(\nu-N_{s}\right) s+i\right)-p_{v}^{n}(i)\right|,
$$

where $p_{v}^{n}(i)$ is the $n$-th-order polynomial fitness check of segment $\nu$.

Step 4. We take the arithmetic average of all the detrended segment variances, which generates the $q$-th order of the fluctuation function. The fluctuation functions are constructed by the values of $q$. The equations are as follows.

If $q \neq 0$,

$$
F_{q}(s)=\left[\frac{1}{2 N_{s}} \sum_{v=1}^{2 N_{s}}\left[F^{2}(v, s)\right]^{q / 2}\right]^{1 / q} .
$$

If $q=0$,

$$
F_{0}(s)=\exp \left[\frac{1}{4 N_{s}} \sum_{v=1}^{2 N_{s}} \ln \left[F^{2}(\nu, s)\right]\right.
$$

Generally speaking, the fluctuation function $F_{q}(s)$ is determined by the time length $s$ under a certain value of $q$. In addition, the fluctuation function equals the traditional detrended cross-correlation analysis process when the value of $q$ is 2 . With this view, we repeat the procedures from Step 2 to Step 4 with various selections of $s$, which is an essential part of the multifractal analysis and, finally, leads to our last procedure, Step 5.

Step 5. Due to the multilength value selection of $s$, the fluctuation function $F_{q}(s)$ with different scaling orders $q$ can be observed by checking the gradient of the log-log plots of $F_{q}(s)$ versus s. If the time series $X(i)$ and $Y(i)$ present a cross-correlation with multifractal property, the fluctuation function $F_{q}(s)$ would demonstrate a power law relationship with a large enough time length $s$ between the two time series: 


$$
F_{q}(s) \sim s^{H_{X Y}(q)},
$$

where $H_{X Y}(q)$ is on behalf of the gradient of the log-log plots of $F_{q}(s)$ with the variation in the values of $q . H_{X Y}(q)$ is estimated by the ordinary least-squares process.

With this view, we find that the slopes of the functions $F_{q}(s)$ and $H_{X Y}(q)$ varied upon the value changes of scaling order $q$. Also, when $q=2, H_{X Y}(q)$ works as the standard Hurst exponent. If the scaling exponent, $H_{X Y}(2)$, is larger than 0.5 , we believe that a persistent cross-correlation exists. However, if the scaling exponent of $H_{X Y}(2)$ is less than 0.5 , we believe that persistent cross-correlation between the time series $X(i)$ and $Y(i)$ does not exist. If the scaling exponent $H_{X Y}(2)$ is equal to 0.5 , the cross-correlation between the time series $X(i)$ and $Y(i)$ shows no significance. With widespread application of the Hurst exponent, $H_{X Y}(2)$ has been commonly viewed as the generalized Hurst exponent. Thus, if the scaling exponent $H_{X Y}(q)$ is equal to a constant by any given value of scaling order $q$, the time series crosscorrelation is believed to hold the monofractal characteristic. In contrast, if $H_{X Y}(q)$ monotonously decreases with the increasing value of $q$, the time series are determined to be cross-correlated with multifractal property. Additionally, we can derive from equations (4) and (6) that when $q$ is greater than zero, the segment $v$, which is on behalf of the large fluctuation of $F^{2}(v, s)$, plays a vital role in valuing fluctuation function $F_{q}(s)$. With this view, the scaling exponent $H_{X Y}(q)$ could be employed as a proxy for the illustration of the large fluctuation scaling character. Conversely, if $q$ is less than zero, the scaling exponent $H_{X Y}(q)$ would be responsible for the small fluctuation scaling character.

\section{Empirical Results}

5.1. Cross-Correlation Test. To have a qualitative view of the cross-correlation between the US dollar exchange rate and US economic policy uncertainty index, we follow the work of Podobnik and Stanley [36] by calculating cross-correlation indicator, $C_{i}$, and statistics, $Q_{\mathrm{cc}}(m)$. Figure 1 demonstrates the cross-correlation statistic $Q_{c c}(m)$ and chi-square critical value $\chi^{2}(m)$ at $5 \%$ significant level with degrees of freedom from 1 to $N-1$. The black and green lines are responsible for the critical value of $\chi^{2}(m)$ and statistic $Q_{c c}(m)$, respectively. We can find that partial $Q_{c c}(m)$ statistics are equal to the critical values; thus, the null hypothesis of no cross-correlations cannot be fully accepted, which would result in a cross-correlation between US dollar exchange rate and US economic policy uncertainty index.

\subsection{Multifractal Detrended Cross-Correlation Analysis.} Section 5.1 provides qualitative evidence that US dollar exchange rate return and US economic policy uncertainty index would have cross-correlation with significant statistics. To have a more solid cross-correlation inspection in a quantitative way, we utilize the prevailing multifractal detrended cross-correlation analysis as the vehicle, which is extensively employed in the time series studies. We calculate the fluctuation function $F_{\mathrm{xyq}}(S)$ ) with growing scaling order

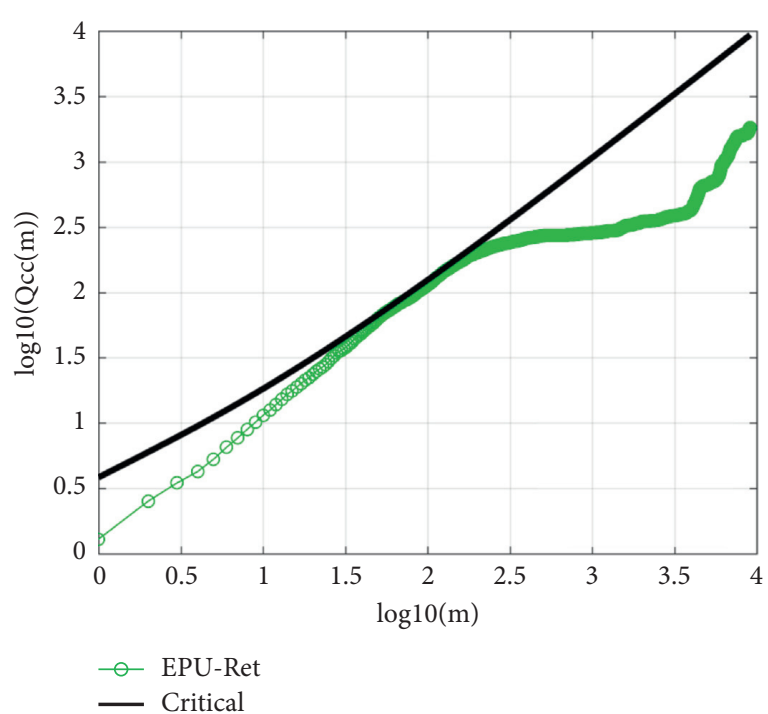

FIgURE 1: Log-log plot of cross-correlation statistic $Q_{\mathrm{cc}}(m)$ for EPU and Ret.

$q$ from -10 to 10 by one step length. Figure 2 plots the log$\log$ trend of $F_{\text {xyq }}(S)$ ) varying upon the time length $s$ between US dollar exchange rate return and US economic policy uncertainty index (Ret and EPU). The lines increasing from bottom to the top are on behalf of corresponding scale orders from -10 to 10 . It is easy to find that $F_{\text {xyq }}(S)$ ) demonstrates apparent rising trend with the gradual increase of $s$ with all scale orders, showing a power law correlation existence within the two time series.

As one of the essential inspection procedures for US dollar exchange rate return and US economic policy uncertainty index cross-correlation check, we calculate the Hurst exponent conditional on scaling order. Figure 3 shows the Hurst exponent evolution pattern upon variation of order $q$. We can find that the Hurst exponent values of US economic policy uncertainty index and US dollar exchange rate return (EPU-Ret) demonstrate downward pattern with increasing scale order. However, all values are greater than 0.5 , indicating cross-correlation persistence between US economic policy uncertainty index and US dollar exchange rate return (EPU-Ret).

In order to have a deep insight of Hurst exponent evolution conditional on the time length $s$, we follow the methodology proposed by Podobnik et al. [38]. Podobnik et al. [38] divided the whole-time length into two parts, the short-term length and long-term length with a cutoff point at $S^{*}$, which indicates a fundamental change in Hurst exponent linear tendency. If the time length $S$ is greater than that of $S^{*}$, we attribute it as long-term length and vice versa. As shown in Figure 2, $\log _{10}\left(S^{*}\right)$ is equal to $2.2\left(S^{*}\right.$ equals 158), marked by the vertical dashed line. Figure 4 presents the short-term and long-term Hurst exponent evolutions. We can find that the long-term Hurst exponents for EPURet are all larger than those in the short term, indicating a more persistent cross-correlation compared with that in the short term. Also, we can find that both long-term and shortterm Hurst exponents decline as scaling order $q$ increases. 


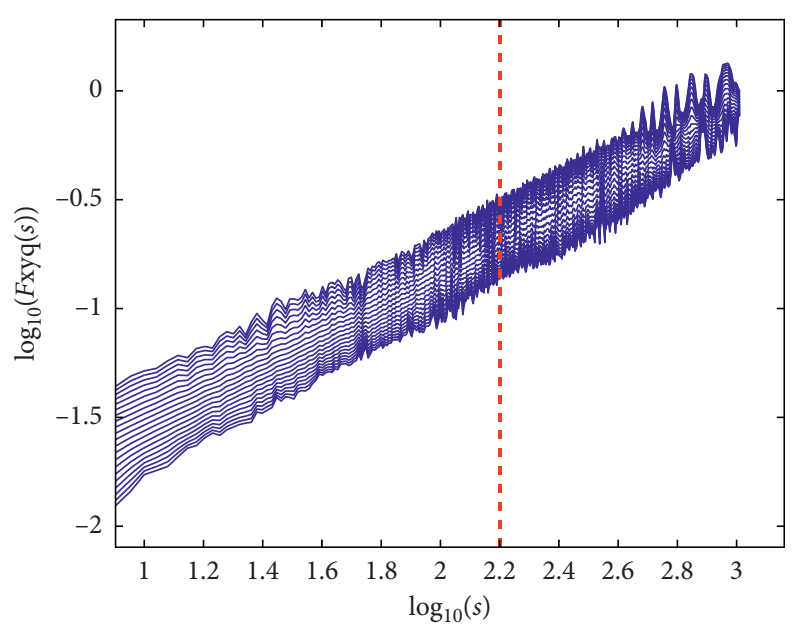

FIGURE 2: Log-log plot of $F_{\mathrm{xyq}}(S)$ versus $s$ for EPU and Ret.

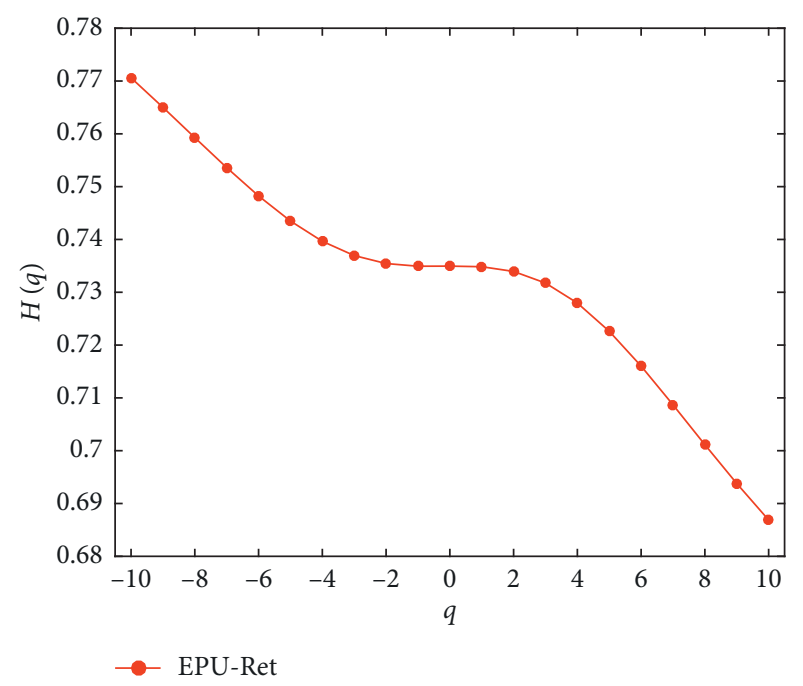

FIgURE 3: Hurst exponent $H(q)$ versus $q$ for the EPU-Ret.

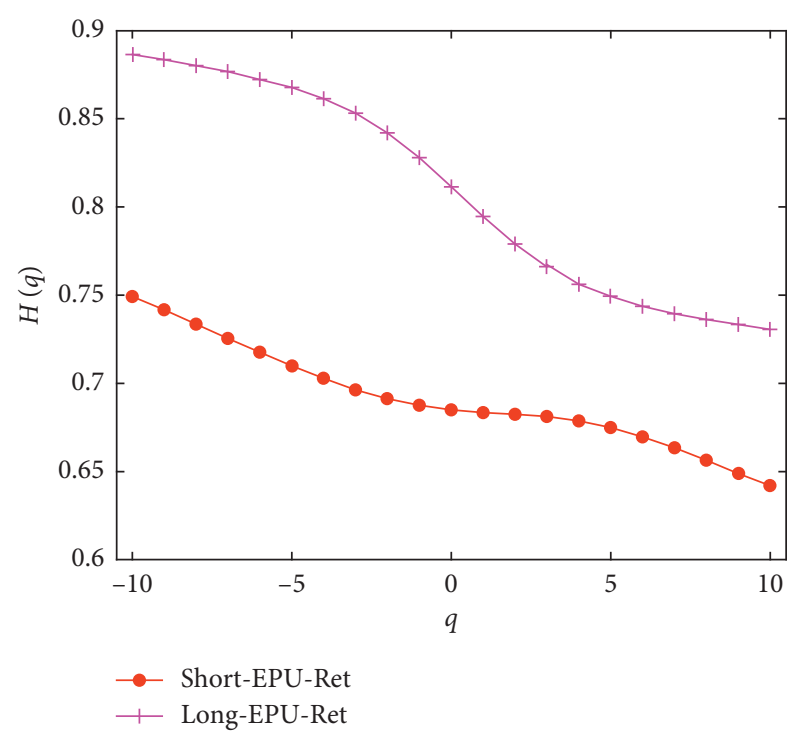

FIgURE 4: Short-term and long-term Hurst exponent $H(q)$ versus $q$ for the EPU-Ret.
With this view, the large fluctuation holds less cross-correlation than the small one. In addition, we introduce $\Delta H_{q}$, the difference between max and $\min \mathrm{H}_{q}$, proposed by Yuan et al. [39], as the measurement instrument of multifractality degree. A smaller value of $\Delta H_{q}$ refers to a lower degree of multifractality. $\Delta H_{q}$ is constructed as follows:

$$
\Delta H_{q}=\max \left(H_{q}\right)-\min \left(H_{q}\right) .
$$

As reported in the last row of Table 2, we can find that long-term $\Delta H_{q}(0.1559)$ is larger than that in the short term (0.1071), indicating a more stable cross-correlation within series.

5.3. Rolling Window Analysis. In this section, we perform the MF-DCCA with rolling window approach to check the cross-correlation dynamic character between US dollar exchange rate return and US economic policy uncertainty index. Due to sample size expansion, we take a four times multiplier of the rolling window setting of Zhang et al. [40] with 2000 trading days as window length and 32, 64, 128, 256,512 , and 1024 as scale $s$. Also, to have a more general view of trend evolution, we follow the work of Zhang et al. [41] with $q$ as 2, 6, and 10. Figure 5 presents the Hurst exponent evolution following rolling window methodology. It is easy to find that the exponent line never moves down 0.5 , showing reliable cross-correlation persistence between EPU and Ret. In addition, we perform two other robustness tests with window sizes of 1500 and 2500 as shown in Figures 6 and 7, respectively. It is easy to find that all the lines are above 0.5 , which are consistent with findings in Figure 5.

5.4. A Binomial Measure from P-Model. Podobnik and Stanley [36] proposed that when scale order $q$ equals 2 , the Hurst exponent within two autoregressive fractional integrated series of same random noise would roughly bear an equivalence to the mean value of the corresponding individual Hurst exponents. Also, Zhou [37] found that if multifractality can be discovered through an iterative way, 
TABLE 2: Short-term and long-term Hurst exponent $H(q)$ versus $q$.

\begin{tabular}{|c|c|c|}
\hline \multirow{3}{*}{$q$} & \multicolumn{2}{|c|}{ EPU-Ret } \\
\hline & \multicolumn{2}{|c|}{$S^{*}=158$} \\
\hline & $S<S^{*}$ & $S>S^{*}$ \\
\hline-10 & 0.7491 & 0.8863 \\
\hline-9 & 0.7415 & 0.8833 \\
\hline-8 & 0.7335 & 0.8801 \\
\hline-7 & 0.7255 & 0.8765 \\
\hline-6 & 0.7174 & 0.8725 \\
\hline-5 & 0.7097 & 0.8676 \\
\hline-4 & 0.7026 & 0.8613 \\
\hline-3 & 0.6963 & 0.8530 \\
\hline-2 & 0.6912 & 0.8420 \\
\hline-1 & 0.6874 & 0.8279 \\
\hline 0 & 0.6848 & 0.8115 \\
\hline 1 & 0.6833 & 0.7945 \\
\hline 2 & 0.6822 & 0.7789 \\
\hline 3 & 0.6809 & 0.7661 \\
\hline 4 & 0.6787 & 0.7563 \\
\hline 5 & 0.6749 & 0.7490 \\
\hline 6 & 0.6697 & 0.7436 \\
\hline 7 & 0.6633 & 0.7394 \\
\hline 8 & 0.6562 & 0.7360 \\
\hline 9 & 0.6490 & 0.7331 \\
\hline 10 & 0.6419 & 0.7305 \\
\hline$\Delta H_{q}$ & 0.1071 & 0.1559 \\
\hline
\end{tabular}

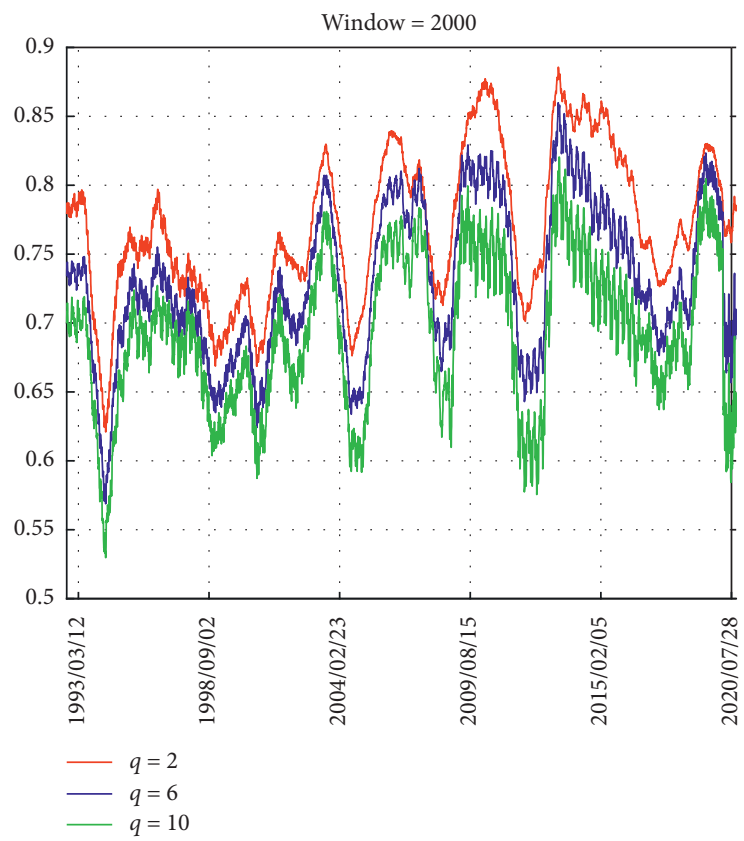

Figure 5: Dynamic Hurst exponents evolution for EPU-Ret $(q=2$, 6 , and 10 , and window $=2000$ ).

the following equation for two binomial measure series would exist:

$$
H_{x y}(q)=\frac{H_{x x}(q)+H_{y y}(q)}{2} .
$$

Figure 8 plots the Hurst exponents evolution with three time series. We can find that the exponents of $H_{\mathrm{xx}}(q)$ and

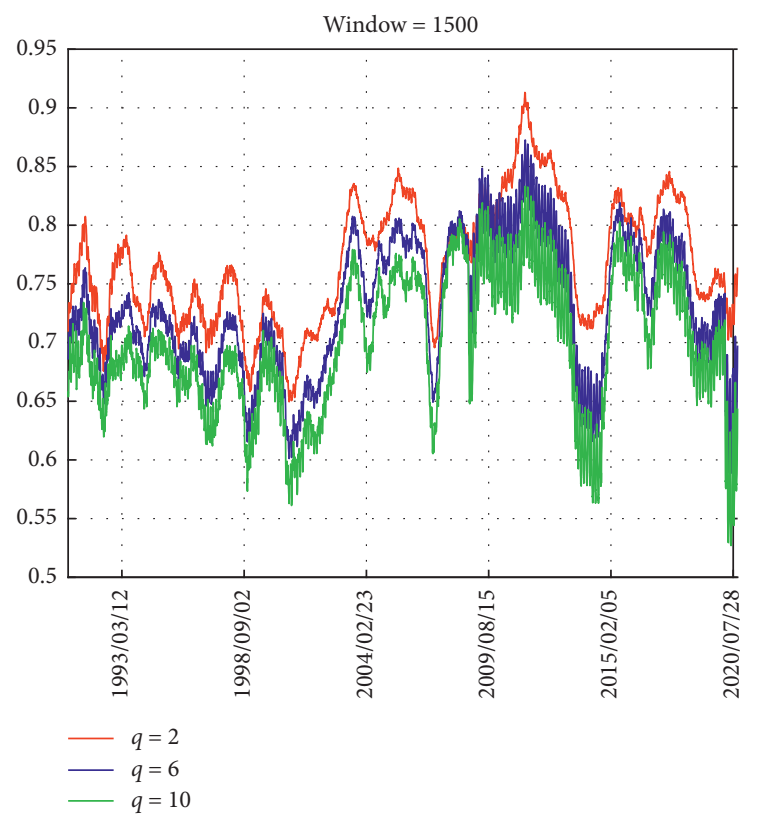

Figure 6: Dynamic Hurst exponents evolution for EPU-Ret $(q=2$, 6 , and 10 , and window $=1500$ ).

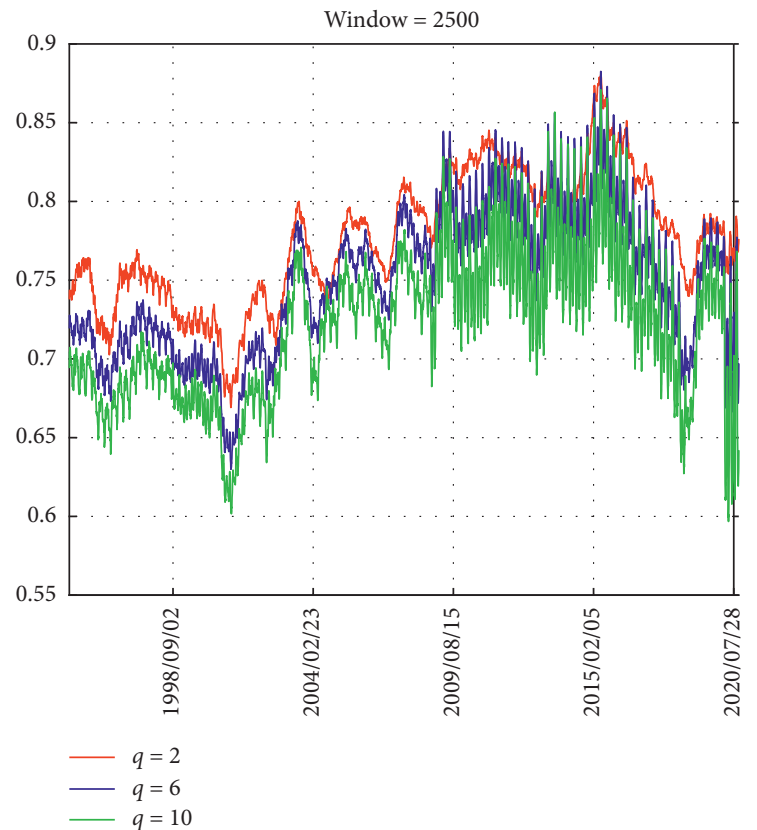

Figure 7: Dynamic Hurst exponents evolution for EPU-Ret $(q=2$, 6 , and 10 , and window $=2500$ ).

$H_{\mathrm{yy}}(q)$ demonstrate downward patterns with reduced values as $q$ increases, indicating multifractality existence of EPU and Ret series. Also, the arithmetic average of $H_{\mathrm{xx}}(q)$ and $H_{\mathrm{yy}}(q)$ presents larger value than that of $H_{\mathrm{xy}}(q)$ as $q$ below 0 and vice versa for $q$ greater than 0 . In addition, the differences between the arithmetic average and $H_{\mathrm{xy}}(q)$ are small and almost symmetrically distributed. Taking this view into consideration, we believe that the differences can be offset and $H_{\mathrm{xy}}(q)$ and $\left(H_{\mathrm{xx}}(q)+H_{\mathrm{yy}}(q)\right) / 2$ are roughly equal within the whole scaling orders. 


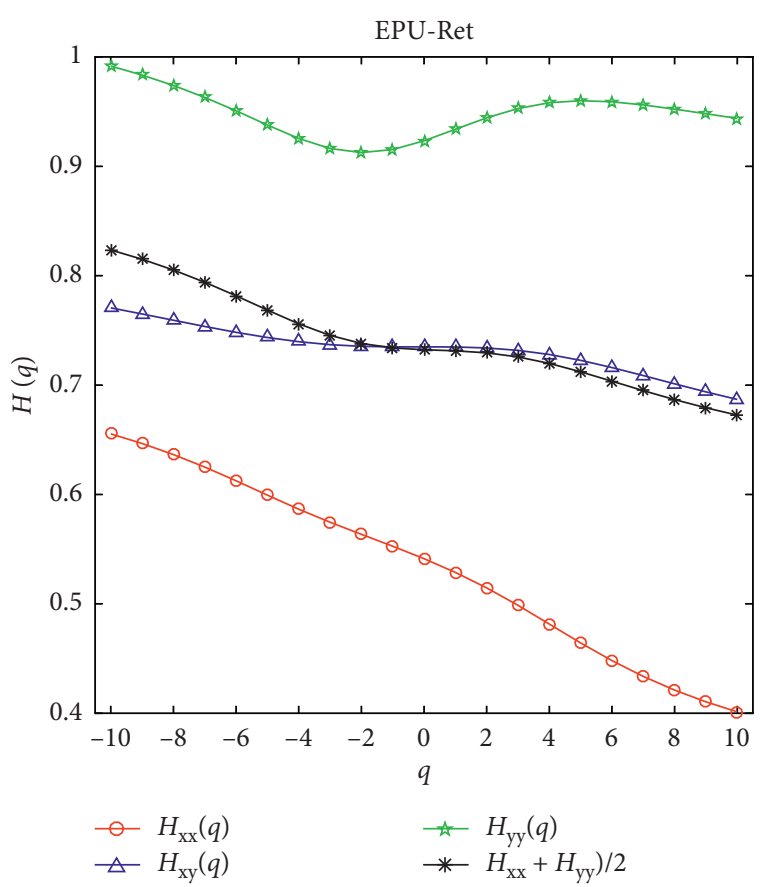

Figure 8: Hurst exponents $H(q)$ versus $q$ for EPU-EPU, EPU-Ret, and Ret-Ret.

\section{Conclusions}

Multifractal detrended cross-correlation analysis has been confirmed as a trustworthy instrument for the detection of cross-correlation multifractality properties between series by many studies in the financial field. With this view, we utilize the fashionable logarithmic US economic policy uncertainty index as the proxy of the US economic policy uncertainty and concatenate it with the matching US dollar exchange rate return. We calculate the cross-correlation statistics and find weak evidence in the existence of cross-correlation between US dollar exchange rate return and US economic policy uncertainty index in the qualitative view. To have more quantitative insight of the multifractal cross-correlation character within series, we employ the MF-DCCA methodology. We find that US dollar exchange rate return and US economic policy uncertainty index present power law cross-correlations with all the scaling orders, confirming the multifractality property between series. Besides, we find that the Hurst exponents between US dollar exchange rate return and US economic policy uncertainty index are all above the critical values with all scaling orders, confirming reliable cross-correlation persistence. Also, the long-term cross-correlation demonstrates more persistence and higher degree of multifractality with larger exponents. Finally, we perform additional rolling window and binomial measure checks. We find that both results are consistent with the model statements.

\section{Data Availability}

The EPU and US dollar index data used to support the findings of this study are included within the article.

\section{Disclosure}

The views expressed in this paper are those of the authors and do not necessarily represent the views of the Shenzhen Stock Exchange.

\section{Conflicts of Interest}

The authors declare that they have no conflicts of interest.

\section{Acknowledgments}

This work was supported by the National Natural Science Foundation of China (71901107, 72001149, and 71790594) and the Fundamental Research Funds for the Central Universities (2019JDZD16).

\section{References}

[1] R. Bansal and A. Yaron, "Risks for the long run: a potential resolution of asset pricing puzzles," The Journal of Finance, vol. 59, no. 4, pp. 1481-1509, 2004.

[2] H. Chen, "Macroeconomic conditions and the puzzles of credit spreads and capital structure," The Journal of Finance, vol. 65, no. 6, pp. 2171-2212, 2010.

[3] H. S. Bhamra, L.-A. Kuehn, and I. A. Strebulaev, "The levered equity risk premium and credit spreads: a unified framework," Review of Financial Studies, vol. 23, no. 2, pp. 645-703, 2010.

[4] S. R. Baker, N. Bloom, and S. J. Davis, "Measuring economic policy uncertainty*," The Quarterly Journal of Economics, vol. 131, no. 4, pp. 1593-1636, 2016.

[5] K. Lee, Y. Jeon, and C. Jo, "Chinese economic policy uncertainty and U.S. households' portfolio decisions," PacificBasin Finance Journal, vol. 64, Article ID 101452, 2020.

[6] N. Attig, S. El Ghoul, O. Guedhami, and X. Zheng, "Dividends and economic policy uncertainty: international evidence," Journal of Corporate Finance, vol. 66, p. 101785, 2020.

[7] F. Li, T. Liang, and H. Zhang, "Does economic policy uncertainty affect cross-border M\&As? - - a data analysis based on Chinese multinational enterprises," International Review of Financial Analysis, vol. 73, p. 101631, 2020.

[8] G. Zhang, J. Han, Z. Pan, and H. Huang, "Economic policy uncertainty and capital structure choice: evidence from China," Economic Systems, vol. 39, no. 3, pp. 439-457, 2015.

[9] E. Demir and O. Ersan, "Economic policy uncertainty and cash holdings: evidence from BRIC countries," Emerging Markets Review, vol. 33, pp. 189-200, 2017.

[10] Y. Zhu, Y. Sun, and X. Xiang, "Economic policy uncertainty and enterprise value: evidence from Chinese listed enterprises," Economic Systems, vol. 39, p. 100831, 2020.

[11] H.-C. Hsieh and V. Q. T. Nguyen, "Economic policy uncertainty and illiquidity return premium," The North American Journal of Economics and Finance, p. 101291, 2020.

[12] R. D'Mello and F. Toscano, "Economic policy uncertainty and short-term financing: the case of trade credit," Journal of Corporate Finance, vol. 64, p. 101686, 2020.

[13] S. Barraza and A. Civelli, "Economic policy uncertainty and the supply of business loans," Journal of Banking \& Finance, vol. 121, p. 105983, 2020.

[14] J. Yang, C. Yang, and X. Hu, "Economic policy uncertainty dispersion and excess returns: evidence from China," Finance Research Letters, p. 101714, 2020. 
[15] X. Wang, W. Xu, and Z. Zhong, "Economic policy uncertainty, CDS spreads, and CDS liquidity provision," Journal of Futures Markets, vol. 39, pp. 461-480, 2019.

[16] G. Çolak, A. Gungoraydinoglu, and Ö. Öztekin, "Global leverage adjustments, uncertainty, and country institutional strength," Journal of Financial Intermediation, vol. 35, pp. 41-56, 2018.

[17] X. Li, "The impact of economic policy uncertainty on insider trades: a cross-country analysis," Journal of Business Research, vol. 119, pp. 41-57, 2020.

[18] X.-M. Li, "New evidence on economic policy uncertainty and equity premium," Pacific-Basin Finance Journal, vol. 46, pp. 41-56, 2017.

[19] B. Lin and R. Bai, "Oil prices and economic policy uncertainty: evidence from global, oil importers, and exporters' perspective," Research in International Business and Finance, vol. 56, p. 101357, 2021.

[20] C. H. J. Cheng, C.-W. Chiu, W. B. Hankins, and A.-L. Stone, "Partisan conflict, policy uncertainty and aggregate corporate cash holdings," Journal of Macroeconomics, vol. 58, pp. 78-90, 2018.

[21] H. Gulen and M. Ion, "Policy uncertainty and corporate investment," The Review of Financial Studies, vol. 29, pp. 523-564, 2016.

[22] P. Wang, X. Li, D. Shen, and W. Zhang, "How does economic policy uncertainty affect the bitcoin market?" Research in International Business and Finance, vol. 53, p. 101234, 2020.

[23] B. B. Mandelbrot and J. W. Van Ness, "Fractional brownian motions, fractional noises and applications," SIAM Review, vol. 10, no. 4, pp. 422-437, 1968.

[24] Q. Ruan, H. Cui, and L. Fan, “China's soybean crush spread: nonlinear analysis based on MF-DCCA," Physica A: Statistical Mechanics and Its Applications, vol. 554, p. 123899, 2020.

[25] Z. Zhang, Y. Zhang, D. Shen, and W. Zhang, "The dynamic cross-correlations between mass media news, new media news, and stock returns," Complexity, vol. 2018, Article ID 7619494, 11 pages, 2018.

[26] X. Xiong, K. Xu, and D. Shen, "Dynamic cross-correlations between investors' attention and CSI300 index futures," Fluctuation and Noise Letters, vol. 18, no. 4, p. 1950022, 2019.

[27] S. Li, X. Lu, and X. Liu, "Dynamic relationship between Chinese RMB exchange rate index and market anxiety: a new perspective based on MF-DCCA," Physica A: Statistical Mechanics and Its Applications, vol. 541, p. 123405, 2020.

[28] Y. Zhou, B. Lu, D. Lv, and Q. Ruan, "The informativeness of options-trading activities: non-linear analysis based on MFDCCA and Granger test," Physica A: Statistical Mechanics and Its Applications, vol. 534, p. 122269, 2019.

[29] Y. Cai, X. Lu, Y. Ren, and L. Qu, "Exploring the dynamic relationship between crude oil price and implied volatility indices: a MF-DCCA approach," Physica A: Statistical Mechanics and Its Applications, vol. 536, p. 120973, 2019.

[30] M. E. Alaoui, E. Bouri, and D. Roubaud, "Bitcoin price-volume: a multifractal cross-correlation approach," Finance Research Letters, vol. 31, 2018.

[31] J. Wang, W. Shao, and J. Kim, "Analysis of the impact of COVID-19 on the correlations between crude oil and agricultural futures," Chaos, Solitons \& Fractals, vol. 136, p. 109896, 2020.

[32] W. Zhang, P. Wang, X. Li, and D. Shen, "Multifractal detrended cross-correlation analysis of the return-volume relationship of bitcoin market," Complexity, vol. 2018, pp. 8691420-20, 2018.
[33] J. W. Kantelhardt, S. A. Zschiegner, E. Koscielny-Bunde, S. Havlin, A. Bunde, and H. E. Stanley, "Multifractal detrended fluctuation analysis of nonstationary time series," Physica A: Statistical Mechanics and Its Applications, vol. 316, no. 1-4, pp. 87-114, 2002.

[34] C.-K. Peng, S. V. Buldyrev, A. L. Goldberger et al., "Longrange correlations in nucleotide sequences," Nature, vol. 356, no. 6365 , pp. 168-170, 1992.

[35] C.-K. Peng, S. V. Buldyrev, S. Havlin, M. Simons, H. E. Stanley, and A. L. Goldberger, "Mosaic organization of DNA nucleotides," Physical Review E, vol. 49, no. 2, pp. 1685-1689, 1994.

[36] B. Podobnik and H. E. Stanley, "Detrended cross-correlation analysis: a new method for analyzing two nonstationary time series," Physical Review Letters, vol. 100, Article ID 084102, 2008.

[37] W.-X. Zhou, "Multifractal detrended cross-correlation analysis for two nonstationary signals," Physical Review E, vol. 77, Article ID 066211, 2008.

[38] B. Podobnik, I. Grosse, D. Horvatić, S. Ilic, P. C. Ivanov, and H. E. Stanley, "Quantifying cross-correlations using local and global detrending approaches," The European Physical Journal $B$, vol. 71, no. 2, p. 243, 2009.

[39] Y. Yuan, X.-t. Zhuang, and X. Jin, "Measuring multifractality of stock price fluctuation using multifractal detrended fluctuation analysis," Physica A: Statistical Mechanics and Its Applications, vol. 388, no. 11, pp. 2189-2197, 2009.

[40] W. Zhang, P. Wang, X. Li, and D. Shen, "Quantifying the cross-correlations between online searches and Bitcoin market," Physica A: Statistical Mechanics and Its Applications, vol. 509, pp. 657-672, 2018.

[41] W. Zhang, Y. Li, Z. Zhang, and D. Shen, "The dynamic crosscorrelations between foreign news, local news and stock returns," Physica A: Statistical Mechanics and Its Applications, vol. 509, pp. 861-872, 2018. 